\title{
Following the trail of source languages in literary translations
}

\author{
Carmen Klaussner, Gerard Lynch and Carl Vogel
}

\begin{abstract}
We build on past research in distinguishing English translations from originally English text, and in guessing the source language where the text is deemed to be a translation. We replicate an extant method in relation to both a reconstruction of the original data set and a fresh data set compiled on an analogous basis. We extend this with an analysis of the features that emerge from the combined data set. Finally, we report on an inverse use of the method, not as guessing the source language of a translated text, but as a tool in quality estimation, marking a text as requiring inspection if it is guessed to be a translation, rather than a text composed originally in the language analysed. With obtain c. $80 \%$ accuracy, comparable to results of earlier work in literary source language guessing - this supports the claim of the method's validity in identifying salient features of source language interference.
\end{abstract}

\section{Introduction}

The present study replicates and extends research reported on by Lynch and Vogel (2012), which sought to identify salient features in texts of translated English that discriminate them from texts originally composed in English. That study focused on an English literary text corpus, containing translations into English from French, German and Russian, as well as non-translated English, and achieved c. $80 \%$ accuracy. The feature set included 'document level' statistics, in addition to token-level distinctive features. By comparison, van Halteren (2008) achieved 86\% accuracy in a similar task with speeches in the EUROPARL corpus, using token-level features.

Carmen Klaussner

CNGL, Trinity College Dublin, Ireland e-mail: klaussnc@tcd.ie

Gerard Lynch

CeADAR, University College Dublin, Ireland e-mail: gerard.lynch@ucd.ie

Carl Vogel

Centre for Computing and Language Studies, Trinity College Dublin, Ireland e-mail: vogel@tcd.ie 
The task in the literary discrimination problem considered by Lynch and Vogel (2012) was to correctly identify the source language (L1) of a text, be it a translation or a text composed originally in English. They explored the classification efficacy of different feature types - document-level statistics, such as average word length or readability metrics, aggregated over the whole text and other features are individuated as n-grams of tokens used in the text, or abstracted as part-of-speech (POS) sequences. ${ }^{1}$ The features identified can be regarded as tangible influences of the source language on the target language texts. Text selection was restricted to those originating in the late $19^{\text {th }}$ to early $20^{\text {th }}$ century as made available through the Project Gutenberg archive. To limit the effect of individual author (or translator) style, each author and translator was only represented once in the corpus, the aim being the detection of features of source-language translation effects rather than fingerprints of individual authors or translators. In the present replication of the task, we test the method's overall validity. For this purpose our data set is independent with respect to internal overlaps of translators and authors with distinct texts from those used in earlier work. We deem demonstration of replicability and extensibility to be an important part of scientific endeavour, and therefore we report our findings here.

Thus, section 2 gives an insight into more intrinsic causes of translation effects and recent work that studied their manifestations in translations. Section 3 describes the current data set and methodology for the experiment. In section 4 , we present our experiments and results on the detection of source languages. Finally, section 5 closes with an in-depth analysis of the prediction properties of identified highly discriminatory attributes and compares to those identified on the basis of the previous study's data set. For the purpose of comparison, we further combine both data sets and subsequently analyse highly characteristic features. As a final exercise in quality estimation, we use salient features to see whether they will lead us to texts that contain irregularities in terms of normal English style.

\section{Motivation \& Previous Research}

The task of predicting the source language of a text by identifying elements that indirectly originated in the source language presupposes translation effects, in this case influence of the source language on the target language. Since translators traditionally translate into their native language, an interesting question to consider is how differences between translated and original language might arise. The act of translation requires fidelity to the source text while maintaining adequacy of representation in the target text (Volansky et al., 2013), and given these two forces pulling in opposite directions, it might be expected that one of these factors will prevail.

Translation studies brought forth the idea of translation universals, properties inherent to the act of translation, such as explicitation (the tendency to render content that is expressed implicitly in the source language more explicitly the target lan-

\footnotetext{
${ }^{1}$ Lexical items that could identify works by topic or theme were discarded to retain and use only features widely applicable.
} 
guage) or simplification (the act of simplifying content in the form of, for instance a less complex sentence structure) that were originally introduced by Blum-Kulka (1986). However, the concept of translation universals remains a controversial issue, partly connected to the difficulty of proving it and thus is constantly challenged by emerging research, as recently by Becher (2010) and by Volansky et al. (2013).

The property of interference, which is by nature a phenomenon specific to language pairs, is simpler to investigate, as irregularities in the target language (L2) can generally be traced back to properties of the source language (L1). Most of these influences are likely to be more abstract, and thus difficult to prevent, as well as being highly dependent on the particular translation pair and therefore not necessarily pertaining to universal translation effects. For instance, a study into bidirectional transfer has shown that one is influenced by a language's conceptual properties regardless of whether it was learned as a first or second language (Degani et al., 2011). ${ }^{2}$

Another issue is related to register interference for pairs of languages that have a similar syntactic realisation of certain items, but which do not share the same register or code relation, where two items in L2 of different register point to one lexical and register representation in L1. For example, in German "Ich bin" can translate to both "I am" and "I'm" in English. Whereas the German non-contracted version can also appear in colloquial speech, in contrast "I am" appears mostly in written text and would be rather marked elsewhere. However, a translator would need to be able to make this distinction (in an abstract way) and decide based on the context rather than only the lexical realisation and similarity to the target language construction. Practically, this is likely to result in more instances of "I am" in the target language. Also, interference may arise due to lack of language competence of the translator, for instance with respect to comprehending metaphors or idiomatic expressions to a degree that would allow rephrasing them appropriately given the cultural context of the target language. Failure of this sort may result in word-byword translations that are structurally and lexically unusual in the target language.

Text classification methods trying to identify specific items of translated text likely originating in the source language have only recently been adopted. Work by Baroni and Bernardini (2006) spearheaded the usage of machine learning techniques to separate translated from non-translated text, in this case based on a large Italian newspaper corpus comprising both original texts and translations. A variety of feature types were explored, the most distinguishing being a combination of lemma and mixed unigrams, bigrams and POS trigrams resulting in $\sim 86 \%$ classification accuracy. It showed that mandatory linguistic features in the source language that are optional in the target language are more likely to be overrepresented in a translation from L1 to L2. In work on the EUROPARL corpus, van Halteren (2008) was able to achieve $87.2 \%$ to $96.7 \%$ accuracy in guessing the source language of speeches, taking into account the signalling strength of individual sequences being present for texts originating in particular languages as well as degrees of over- or under-use. A number of studies explored translation direction and translationese,

\footnotetext{
${ }^{2}$ Consequently, both L1 and L2 speakers of Hebrew and English rated word pairs in English closer when they shared a translation in Hebrew, e.g. tool and dish both translate into kli. English monolinguals did not perceive this relatedness of the same word pair.
} 
focused on machine translation. Kurokawa et al. (2009) investigated translation direction in the parallel Canadian Hansard FR-EN corpora and discovered that using FR-EN corpora for a FR-EN machine translation system resulted in the same accuracy (BLEU score) as a much larger corpus of EN-FR. Lembersky et al. (2011) extended this using both the EUROPARL and the Canadian Hansard corpus, focusing in the first instance on the language model used in machine translation, not the phrase tables themselves as done by Kurokawa et al. (2009). Using the perplexity metric on the EUROPARL data, they find that a language model made up of language translated from the source language of the text to be translated had the highest perplexity score, with the worst perplexity score derived from the original English data. A mixed model containing text from all four languages plus original English performed second best. They claim this validates the convergence universal of translation: a mixed translationese model was still a better fit than original English; translationese is more similar to other translationese from different source languages than original English. Lembersky et al. (2012) focused on the phrase tables themselves (like Kurokawa et al. (2009)), replicating the earlier results, delving further into the explanation of why translation direction matters. Using the informationtheoretic metrics of entropy and cross entropy, they find that phrase tables trained in the correct direction contain more unique source phrases and less translations per source phrase, which results in a lower entropy and cross entropy score on the data.

Koppel and Ordan (2011) investigated dialects of translationese, which concerned the different subsets of translations in a target language from numerous source languages. Their work used the frequencies of the top three hundred most frequent words and SVM classifiers and obtained 90\% classification accuracy between translations into English from Hebrew, Korean and Greek from the International Herald Tribune (IHT). They replicated and extended the work on EUROPARL from van Halteren (2008) and reported that results on corpora of same-language translations and original English are generally better than training a translationese detection system on one corpus (EUROPARL) and testing on another (IHT), which returned poor results $(50-60 \%)$, showing that features of translationese vary not only by source language, but also genre. Ilisei et al. (2010) examined translation universals of simplification using document-level metrics only, and reported high accuracy (80-90\%) on two separate corpora of medical and technical translations from Spanish to English. Distinguishing features included lexical richness, the ratio of closed-class to open-class words and the ratio of numerals, adjective and finite verbs, each to total words. This work motivated the inclusion of 'document-level' features in the original study on source language detection by Lynch and Vogel (2012), which is continued here. Similar methods are used in Lynch (2014) for the task of distinguishing authorial style in a corpus of literary translations of three Russian authors by the same translator, distinguishing reliably between authors using document-statistics alone. Forsyth and Lam (2014) also investigates authorial style in parallel translations of Van Gogh's letters using clustering techniques and finds that authorial discriminability is stronger than translatorial discriminability.

Volansky et al. (2013) tested translation hypotheses, such as simplification, explicitation and interference, using supervised machine learning in English with lin- 
guistically informed features in a text classification setting. They tentatively conclude that claims to universal translation effects should be reconsidered, as they not only rely on genre and register, but also vary greatly depending on language pairings. Interference yields the highest performing and most straightforwardly interpretable distinguishing features ("source language shining through"). In the present study, we attempt to identify salient features of interference that originated in the source language, but are not translator-specific issues; i.e. features detected should be general enough to appear across different translations of the same source language and thus signal more intrinsic transfer issues for that particular language pair.

\section{Methods}

First, we replicated the study of Lynch and Vogel (2012), finding results that agree with the earlier work. ${ }^{3}$ Below we describe our methods in extending this method to a new set of literary translations. We used the same restrictions introduced by the earlier study. The data selection process and preprocessing are described in section 3.1; feature sets, in section 3.2; classification methods, in section 3.3.

\subsection{Corpus Selection \& Preparation}

In order to have a comparable data sample with respect to the first analysis, the present set was selected according to the same criteria of being available in machinereadable format and being in the public domain. ${ }^{4}$ Each author should have a unique text and no translators should be repeated in the case of translations. Additionally, all texts should be of sufficient length, meaning at least 200 kilobytes in size.

Since this is the second experiment using this configuration, to be able to generalise better about the model's validity, there should be little or if possible no overlap in authors/translators with the first data set. While being able to meet all other requirements, the last one proved to be more difficult with respect to the Russian corpus, as there were fewer texts available (not theoretically, but practically, since new books are still in the process of being added) and after discarding all those not suited for other reasons, there remained one overlap with the first data set for the translator, Constance Garnett. Generally, one of the objectives was also to not have too large a time-frame, i.e. have all novels written and translated within 100 years, so as to limit differences in style with respect to diachronic effects. It would also have been more desirable for the corpus to contain only novels, whereas in the current set, there is one collection of short stories in the Russian set, namely "The wife

\footnotetext{
${ }^{3}$ See previous and replicated results in table 8 and 9 in the online appendix to this paper: https://www.scss.tcd.ie/ vogel/SGAI2014/KlaussnerLynchVogel-Appendix-SGAI2014.pdf

${ }^{4}$ All were taken from Project Gutenberg: http://www.gutenberg.org/ - last verified Nov. 2013.
} 
Table 1 Text corpora for current source language detection experiment.

\begin{tabular}{|c|c|c|c|c|c|}
\hline Title & Author & Source & Pub. & Translator & Pub. \\
\hline Vanity Fair & William M. Thackeray & English & 1848 & $\mathrm{n} / \mathrm{a}$ & $\mathrm{n} / \mathrm{a}$ \\
\hline Wives and Daughters & Elizabeth Gaskell & English & 1866 & $\mathrm{n} / \mathrm{a}$ & $\mathrm{n} / \mathrm{a}$ \\
\hline The Moonstone & Wilkie Collins & English & 1868 & $\mathrm{n} / \mathrm{a}$ & $\mathrm{n} / \mathrm{a}$ \\
\hline Sylvie and Bruno & Lewis Carroll & English & 1889 & $\mathrm{n} / \mathrm{a}$ & $\mathrm{n} / \mathrm{a}$ \\
\hline The Return of Sherlock Holmes & Arthur Conan Doyle & English & 1904 & $\mathrm{n} / \mathrm{a}$ & $\mathrm{n} / \mathrm{a}$ \\
\hline House of Mirth & Edith Wharton & English & 1905 & $\mathrm{n} / \mathrm{a}$ & $\mathrm{n} / \mathrm{a}$ \\
\hline Only a girl: or, A Physician for the Soul & Wilhelmine von Hillern & German & 1869 & A. L. Wister & 1870 \\
\hline Villa Eden & Berthold Auerbach & German & 1869 & Charles C. Shackford & 1871 \\
\hline The Northern Light & E. Werner & German & 1890 & D.M. Lowrey & 1891 \\
\hline Cleopatra-Complete & Georg Ebers & German & 1893 & Mary J. Safford & 1894 \\
\hline Dame Care & Hermann Sudermann & German & 1887 & Bertha Overbeck & 1891 \\
\hline Royal Highness & Thomas Mann & German & 1909 & A. Cecil Curtis & 1916 \\
\hline The King of the Mountains & Edmond About & French & 1856 & Mrs. C. A. Kingsbury & 1897 \\
\hline Against The Grain & Joris-Karl Huysmans & French & 1884 & John Howard & 1924 \\
\hline The Dream & Emile Zola & French & 1888 & Eliza E. Chase & 1893 \\
\hline Pierre and Jean & Guy de Maupassant & French & 1888 & Clara Bell & 1890 \\
\hline Arsne Lupin versus Herlock Sholmes & Maurice Leblanc & French & 1908 & George Moorehead & 1910 \\
\hline The Gods are Athirst & Anatole France & French & 1912 & Mrs. Wilfrid Jackson & 1913 \\
\hline Dead Souls & Nicholas Gogol & Russian & 1840 & D. J. Hogarth & 1846 \\
\hline The Wife, and Other Stories & Anton Chekhov & Russian & 1892 & Constance Garnett & 1918 \\
\hline The Daughter of the Commandant & Aleksandr S. Pushkin & Russian & 1836 & Mrs. Milne Home & 1891 \\
\hline The Precipice & Ivan Goncharov & Russian & 1869 & unknown/ M. Bryant & 1916 \\
\hline A Russian Gentleman & Sergei T. Aksakov & Russian & 1858 & J.D. Duff & 1917 \\
\hline Satan's Diary & Leonid Andreyev & Russian & 1919 & Herman Bernstein & 1920 \\
\hline
\end{tabular}

and other stories" rather than a continuous text. The difference in genre may affect the distribution of proper nouns, since there are likely to be more different ones.

In the original study, there were five novels per source language, while for ours, one more novel per source language corpus is added, as shown in table 1 . We retain the same file sizes and partition scheme, whereby 200 kilobytes of text are randomly extracted from each novel and divided into pieces of 10 kilobytes each, resulting in 120 text chunks per source language. Limited availability of texts translated from the chosen languages, as well as our constraints on novel size and non-overlap with the previous experiment rendered a significantly larger data set infeasible.

\subsection{Feature Sets}

We consider the same set of document-level features as Lynch and Vogel (2012), shown in table 2 , the inclusion of which had originally been motivated by the exploration of Ilisei et al. (2010) into features other than n-grams. In addition, we selected both part-of-speech (POS) trigrams (including all punctuation) and word unigrams. The previous study used POS bigrams but not POS trigrams. Another alteration in our system is the weighting scheme. In the previous study lexical and POS features were binarized: a feature was observed as either present or not regardless of the exact frequency magnitude. We adopt relative frequency weighting, which in this case is reduced to taking raw frequency counts, since all text chunks have about the same 
Following the trail of source languages in literary translations

size and there is no need to normalise over document length. Binarized weighting was abandoned because of a considerable drop in performance on the current data set, while tests using relative frequency were successful on both data sets.

Table 2 Document level features

\begin{tabular}{llr}
\hline No & \multicolumn{1}{c}{ Feature } & Description \\
\hline 1 & Avgsent & Average sentence length \\
2 & Avgwordlength & $\begin{array}{l}\text { Average word length } \\
\text { Readability metric }\end{array}$ \\
3 & ARI & Readability metric \\
4 & CLI & Ratio Description \\
\hline No & \multicolumn{1}{c}{ Feature } & word types : total words \\
\hline 5 & Typetoken & numerals : total words \\
6 & Numratio & finite verbs : total words \\
7 & Fverbratio & prepositions : total words \\
8 & Prepratio & conjunctions : total words \\
9 & Conjratio & open-class words : total words \\
10 & Infoload & nouns : total words \\
11 & dmarkratio & discourse markers : total words \\
12 & Nounratio & open-class words : closed-class words \\
13 & Grammlex & simple sentences : complex sentences \\
14 & simplecomplex & pronouns : total words \\
15 & Pnounratio & lemmas : total words \\
16 & lexrichness & simple sentences : total sentences \\
17 & simpletotal & complex sentences : total sentences \\
18 & complextotal & complom
\end{tabular}

Table 3 Top 20 ranked features selected for run 8-13.

\begin{tabular}{lr}
\hline Top 20 features & $\chi^{2}$ \\
\hline it's & 53.13 \\
NP NP POS & 52.35 \\
IN NP NP & 50.12 \\
, VBN IN & 46.79 \\
, DT NN & 43.26 \\
, DT JJ & 43.68 \\
don't & 43.56 \\
got & 43.46 \\
DT NNS IN & 43.40 \\
and & 42.63 \\
NNS, DT & 42.31 \\
VBP JJ PP & 41.70 \\
suppose & 40.38 \\
NNS IN DT & 40.35 \\
i'll & 40.11 \\
IN NN , & 39.40 \\
NN, VBN & 39.23 \\
NN, DT & 38.79 \\
Typetoken & 38.77 \\
NP NP SENT & 38.54 \\
\hline
\end{tabular}

\subsection{Classification \& Selection Methods}

For processing of all features, we use $\mathrm{R}$ scripts dealing separately with word unigrams, POS n-grams and document-level features. POS tagging of the text is done using the R koRpus package for POS tagging (that uses TreeTagger POS tagger (Michalke, 2013; Schmid, 1994)). For both POS trigrams and word unigrams, we only retain the most frequent ones to dispose of highly novel-specific proper nouns and terms, as well as thus ensuring that we build a very general classifier.

The process of feature selection and classification remains the same as in the previous study. In order to preselect characteristic features from both the word unigram and POS trigram list, we rank each group separately according to classification power using the $\chi^{2}$ metric, where a higher value indicates a greater divergence over different classes. We retain the most discriminatory 100 POS trigrams and 50 word unigrams. All 18 document-level features, shown in table 2, enter the final selection. 
For machine-learning, we also used Weka (Hall et al., 2009), specifically the Naive Bayes classifier, the SMO classifier, which is an implementation of a Support Vector Machine (SVM) classifier and the Simple Logistic classifier.

\section{Experiments}

\subsection{Method}

For benefit of comparison, experiments are repeated using the same general settings as in the previous study by testing document-level features on their own and having two different configurations for selecting the highest discriminatory features from the preselected features (out of the two $\mathrm{n}$-gram feature groups). In the $1^{\text {st }}$ setting, we classify on only the 18 document-level statistics. For the $2^{\text {nd }}$ setting, we select the best 50 features out of the preselected 100 best POS trigrams, 50 best unigrams and all document-level features, and for the $3^{\text {rd }}$ configuration, rather than 50, we retain only the best 30 features according to $\chi^{2}$ for the final model. The training and test sets are constructed by randomly extracting $\sim 20 \%$ from the full set, resulting in a division of 390 instances for training and 90 instances for evaluation randomly spread over all four classes, which increases the proportion of test items in the total set by $3 \%$ compared to what was used previously. Using only the training data, we execute ten-fold cross validation; we apply separate evaluation against the test set.

\subsection{Results}

Table 4 shows the results for all experiments, using both test set and cross-validation for evaluation. In relation to the first study, results for document-level features in run 2-7 are occasionally both higher and lower. Similarly for the different configurations in runs $8-13$ and runs $14-19$, results move in the same range between $\sim 60 \%$ to $\sim 85 \%$ accuracy. Our highest value, also run 13 is slightly lower, but differences in terms of data set and training and test division probably account for that. Table 3 shows the first 20 features selected for run 8-13 (the full list is shown in table 11 of the online appendix - see fn. 3). In comparison to the earlier study, it is notable that the $\chi^{2}$ value for the most discriminatory features is with 53.13 less than three times as low as the best in the previous study ( $\chi^{2}$ of 191.1184). Another general difference is the composition of the best 50 features. Although the same number of each feature type was considered, our set has a higher number of POS features and fewer unigrams (13), whereas in the previous study, 32 features out of 50 were word unigrams. This difference might be due to our discarding rather infrequent unigrams and thus not considering the complete set of features for preselection. However, it could also be due to POS trigrams (including punctuation) being better discriminators than POS 
Following the trail of source languages in literary translations

bigrams, at least on the current data set. For a clearer analysis, we need to examine the features identified on the previous data set to determine consistent features over both data sets and therefore the ones that are more prevalent for a particular class.

Table 4 Results for model evaluation based on three different sets of features.

\begin{tabular}{|c|c|c|c|c|c|}
\hline Run & Training & Test & Classifier & Features & Accuracy \\
\hline 1 & Full & $10-\mathrm{f}-\mathrm{cv}$ & Baseline & $\mathrm{n} / \mathrm{a}$ & $25.0 \%$ \\
\hline 2 & Full & Test & NaiveBayes & 18 doc-level & $51.8 \%$ \\
\hline 3 & Full & Test & SVM (SMO) & 18 doc-level & $63.0 \%$ \\
\hline 4 & Full & Test & SimpLog & 18 doc-level & $67.9 \%$ \\
\hline 5 & Full & $10-\mathrm{f}-\mathrm{cv}$ & NaiveBayes & 18 doc-level & $56.2 \%$ \\
\hline 6 & Full & $10-\mathrm{f}-\mathrm{cv}$ & SVM (SMO) & 18 doc-level & $60.2 \%$ \\
\hline 7 & Full & $10-\mathrm{f}-\mathrm{cv}$ & SimpLog & 18 doc-level & $65.3 \%$ \\
\hline 8 & Full & Test & NaiveBayes & Top50(100POStri+18doc+50wuni) & $73.8 \%$ \\
\hline 9 & Full & Test & SVM (SMO) & Top50(100POStri+18doc+50wuni) & $79.7 \%$ \\
\hline 10 & Full & Test & SimpLog & Top50(100POStri+18doc+50wuni) & $76.9 \%$ \\
\hline 11 & Full & $10-\mathrm{f}-\mathrm{cv}$ & NaiveBayes & Top50(100POStri+18doc+50wuni) & $77.2 \%$ \\
\hline 12 & Full & $10-\mathrm{f}-\mathrm{cv}$ & SVM (SMO) & Top50(100POStri+18doc+50wuni) & $82.7 \%$ \\
\hline 13 & Full & $10-\mathrm{f}-\mathrm{cv}$ & SimpLog & Top50(100POStri+18doc+50wuni) & $84.5 \%$ \\
\hline 14 & Full & Test & NaiveBayes & Top30(100POStri+18doc+50wuni) & $59.4 \%$ \\
\hline 15 & Full & Test & SVM (SMO) & Top30(100POStri+18doc+50wuni) & $76.2 \%$ \\
\hline 16 & Full & Test & SimpLog & Top30(100POStri+18doc+50wuni) & $71.1 \%$ \\
\hline 17 & Full & $10-\mathrm{f}-\mathrm{cv}$ & NaiveBayes & Top30(100POStri+18doc+50wuni) & $69.7 \%$ \\
\hline 18 & Full & $10-\mathrm{f}-\mathrm{cv}$ & SVM (SMO) & Top30(100POStri+18doc+50wuni) & $77.7 \%$ \\
\hline 19 & Full & $10-\mathrm{f}-\mathrm{cv}$ & SimpLog & Top30(100POStri+18doc+50wuni) & $74.5 \%$ \\
\hline
\end{tabular}

\section{Analysing Discriminatory Features}

In this section, we explore salient features of this study, and the features obtained when we combine the two data sets, with respect to what they predict for the source languages. We close with an exercise in quality estimation using salient features.

\subsection{Feature Prediction}

To determine what features predict in terms of the four classes, we analyse those prominent 50 features that emerged from this study as being best in discriminating between classes. If a feature is frequent in a particular translation, but not in the English corpus, this might signal potential source language interference. We again 
Table 5 Feature proportions over entire source language corpus. Bold features are those significantly more frequent and Italic features and those significantly less frequent than expected with respect to the feature type distribution over that source language.

\begin{tabular}{|c|c|c|c|c|}
\hline \multicolumn{5}{|c|}{ Ranked Features $\%$ English $\%$ French $\%$ German $\%$ Russian } \\
\hline it's & 61 & 3 & 19 & 17 \\
\hline NP NP POS & 65 & 9 & 17 & 9 \\
\hline IN NP NP & 48 & 17 & 16 & 18 \\
\hline , VBN IN & 15 & 46 & 19 & 20 \\
\hline , DT NN & 17 & 37 & 22 & 24 \\
\hline , DT JJ & 16 & 40 & 21 & 23 \\
\hline don't & 42 & 9 & 16 & 33 \\
\hline got & 48 & 10 & 17 & 25 \\
\hline DT NNS IN & 19 & 34 & 25 & 22 \\
\hline and & 23 & 23 & 25 & 29 \\
\hline NNS, DT & 13 & 48 & 17 & 23 \\
\hline VBP JJ PP & 64 & 6 & 16 & 14 \\
\hline suppose & 59 & 10 & 15 & 16 \\
\hline NNS IN DT & 21 & 35 & 23 & 21 \\
\hline i'll & 58 & 0 & 27 & 14 \\
\hline IN NN, & 20 & 29 & 24 & 27 \\
\hline $\mathrm{NN}, \mathrm{VBN}$ & 15 & 46 & 19 & 20 \\
\hline $\mathrm{NN}, \mathrm{DT}$ & 14 & 40 & 22 & 24 \\
\hline
\end{tabular}

extracted a sample and analysed each feature in all four source languages by taking the raw counts of the feature in the language and normalising it by the total count for its feature type in the language. ${ }^{5}$ Table 5 shows the proportion of use for each source language in relation to the other languages for 18 of the 50 best features (table 10 in the online appendix contains the complete list—see fn. 3). ${ }^{6}$ Most interesting are those where translations deviate vastly from the English source corpus, e.g. IT's and I'LL-most uses of these items are in the English sample (61\% and 58\%, respectively) and least are in the French source corpus (3\% and 0\%, resp.). Differences in syntactic sequences can be seen for $\langle$ NP NP POS $\rangle,{ }^{7}$ a frequent sequence in English which appears less frequently in translations, or $\langle$ NNS , DT $\rangle$, which is less common in originally composed English than in translations, especially from French.

\subsubsection{Comparison Between Data Sets}

To compare the two data sets ("A" for previous data and "B" for current) on equal terms, we consider the features emerging from only our system. Shared items among the top 50 features selected from each are indicated in table 6 . That they are the most robust features over both sets indicates that they have strongest predictive power. However, there might be differences in what exactly they are predicting between

${ }^{5}$ A POS trigram, e.g. $\langle$ NP NP NP , is relativized to the total number of witnessed POS trigrams.

${ }^{6}$ All proportions are rounded to the nearest integer.

${ }^{7}$ In this context, $\langle\mathrm{POS}\rangle$ refers to "possessive ending" rather than part-of-speech (POS). 
the data sets, and thus we need to look the distributions of the individual features in each set separately. Table 6 shows the raw counts for each feature and the total count of observed feature type (unigrams/POS trigrams). For each feature, the table also shows the percentage of its occurrences within texts of each language. ${ }^{8}$ Consider $\langle$ DT NNS IN $\rangle$ and $\langle$, DT NN $\rangle$, both relatively consistent in use between data sets. The use in English, German and Russian as source language is lower, and in French, higher than would expected if the feature did not depend on source language (with significant Pearson residuals, $p<0.001$ ). Thus, these features are potentially reliable interference signals. However, other features have distributions that differ significantly between data sets. Consider IT's, for example: the proportion in B for English is much greater than in A (61\% vs. $28 \%$ ), where the "Russian" sample has $57 \%$ of the instances of IT's. A $\chi^{2}$ comparison of corresponding tables of counts shows significant difference $(p<0.001)$, with significant Pearson residuals indicating that the frequency of IT's for English in set B and Russian in set A lies well over expected counts for each. Even though this feature is discriminatory for both sets, in A it is indicative of translation from Russian and in B is diagnostic of English original texts. Thus, finding discriminatory features may be easier than finding true signals of source language interference. That candidate interference signals are not reliably diagnostic across data sets is revealed by replication studies like this.

Table 6 Comparison between prediction direction of shared features of both data sets by looking at proportion in $\%$ and raw counts \# of shared items in both sets separately. The left and right table contain proportions of data set A and B respectively. Bold and Italic are used as in Table 5.

\begin{tabular}{|c|c|c|c|c|c|c|c|c|c|c|c|c|c|c|c|c|}
\hline \multirow[b]{2}{*}{ Features } & \multicolumn{8}{|c|}{ Data set A } & \multicolumn{8}{|c|}{ Data set B } \\
\hline & \multicolumn{2}{|c|}{$\begin{array}{l}\text { Eng } \\
\%(\#)\end{array}$} & $\begin{array}{l}\mathrm{Fr} \\
\%\end{array}$ & (\#) & \multicolumn{2}{|l|}{ Ger } & \multicolumn{2}{|l|}{$\mathrm{Ru}$} & \multicolumn{2}{|c|}{ Eng } & $\begin{array}{l}\mathrm{Fr} \\
\%\end{array}$ & (\#) & $\begin{array}{l}\text { Ger } \\
\%\end{array}$ & & $\mathrm{Ru}$ & (\#) \\
\hline it's & 28 & (82) & 6 & (16) & 9 & (27) & 57 & (163) & 61 & (218) & 3 & (10) & 19 & (68) & 17 & (59) \\
\hline don't & 43 & (386) & 5 & (46) & 23 & (205) & 30 & $(270)$ & 42 & (286) & 9 & (58) & 16 & (109) & 33 & (221) \\
\hline , DT NN & 20 & (302) & 38 & (556) & 22 & (330) & 20 & (291) & 17 & (320) & 37 & (697) & 22 & (402) & 24 & (441) \\
\hline DT NNS IN & 23 & (432) & 35 & (663) & 22 & (422) & 20 & (368) & 19 & (437) & 34 & (780) & 25 & (563) & 22 & (515) \\
\hline which & 21 & (582) & 36 & (1011) & 24 & $(680)$ & 18 & $(505)$ & 24 & (1051) & 30 & (1286) & 28 & (1222) & 18 & (771) \\
\hline NP NP NP & 21 & (163) & 50 & (383) & 19 & (144) & 11 & (82) & 39 & (280) & 24 & (171) & 29 & (206) & 9 & (62) \\
\hline that's & 33 & (96) & 5 & (14) & 11 & (31) & 52 & (153) & 48 & (105) & 3 & (7) & 32 & (71) & 17 & (38) \\
\hline the & 23 & (9655) & 30 & (12262) & 25 & (10419) & 22 & (9176) & 24 & (12296) & 27 & (13869) & 26 & (13131) & 22 & (11286) \\
\hline and & 27 & (6363) & 20 & (4866) & 27 & (6473) & 26 & (6092) & 23 & (6526) & 23 & (6466) & 25 & (6944) & 29 & (8179) \\
\hline NN : SENT & 12 & (6) & 2 & (1) & 0 & (0) & 85 & (41) & 3 & (5) & 29 & $(51)$ & 16 & (28) & 53 & (93) \\
\hline $\begin{array}{l}\text { Type totals } \\
\text { unigram }\end{array}$ & & 47912 & & 146380 & & 50362 & & 47637 & & 74428 & & 172429 & & 77486 & & 73300 \\
\hline POS trigram & & 28923 & & 223340 & & 30062 & & 26778 & & 68539 & & 260359 & & 62624 & & 64828 \\
\hline
\end{tabular}

\subsubsection{Combined Data Set}

As a final exercise, we combine both data sets into one, by discarding one novel per class in data set B in order to create a balanced set with respect to the two different

\footnotetext{
${ }^{8}$ This is relevant, since there are four more data samples in set B.
} 
Table 718 most discriminatory features in the combined data set.

\begin{tabular}{lrlrll|lrr}
\hline Sequence & $\chi^{2}$ & Data|Sequence & \multicolumn{2}{c}{$\chi^{2}$} & Data|Sequence & $\chi^{2}$ & Data \\
\hline NP NP POS & 41.27 & B & and & 34.55 & S & NN , DT & 33.39 & B \\
don't & 40.69 & S & SENT NN VBZ & 34.07 & A & CD NNS , & 32.89 & A \\
IN NP NP & 38.11 & B & SENT N N & 33.90 & A & SENT NNS VBP & 32.07 & A \\
, VBN IN & 38.01 & B & suppose & 33.90 & B & Conjratio & 31.98 & S \\
SENT CC PP & 36.30 & A & Nounratio & 33.67 & A & Avgwordlength & 31.93 & A \\
DT NN & 34.92 & S & which & 33.43 & S & though & 31.90 & A \\
\hline
\end{tabular}

sets, i.e. have 5 novels per class per data set, thus 400 instances for each data set and 800 in total. We apply the settings from the most successful runs/configurations, $11-13$, by preselecting 168 features out of 50 word unigrams, 100 POS trigrams and 18 document-level features and then again selecting the best 50 out of those using $\chi^{2}$. The results although slightly lower than what was achieved earlier are still well in the range of the previous one (c. $75 \%$ to $82 \%$ ). In table 7 the "Data" column indicates if the feature was prevalent in data set $\mathbf{A}, \mathbf{B}$ or $\mathbf{S}$ if it was shared.

\subsection{An Exercise in Quality Estimation}

We have identified features in translated texts likely to have originated in the source language. However, since these were selected on the basis of a statistical optimisation, it is not guaranteed that texts containing these features would be judged equally anomalous by native speakers of English. Here, we examine whether highly salient features of our model index sentences that a human would strongly perceive as either original English or translated English. We selected a number of salient features for each source language and sentences in which they occur from the data set.

The gold standard notes whether an item was a translated text or originally composed in English. We obtained two human judgements on 17 sentences selected across our corpus. ${ }^{9}$ The sentences were selected manually to maximise features distinctive for the source languages. The kappa statistic computed between the raters' judgements is 0.406 , suggesting moderate agreement. On 7 out of 17 instances, both raters agreed with the gold standard and on another 5 , at least one was correct. ${ }^{10}$

Some sentences were identified by both annotators as translations, as for instance example (1), which has feature type $\langle, \mathrm{CC}$ RB $\rangle$ of German English. There is some disagreement between other ratings of translated pieces, but interestingly, not only the translated sentences introduced confusion: example (2) is an instance of original English (tagged to include $\langle$ IN NP NP $\rangle$ ), but was judged translated by both raters.

\footnotetext{
${ }^{9}$ The assessors are native English speakers among co-authors of this paper.

${ }^{10}$ On 5 items, both raters agreed with each other but not with the gold standard: in 3 of those the source was English, and both raters judged the item odd; in the other 2, the source was not English.
} 
(1) ... whereupon the Professor [...] had then expressed his thanks with glad surprise, and indeed emotion, for the kind interest the Prince had expressed in his lectures.

(2) The young couple had a house near Berkeley Square and a small villa at Roehampton, among the banking colony there.

This evidence constitutes anecdotal support for the claim that the method is correctly discriminative: items that are high in distinctive features that would be identified by the system slip by human scrutiny in $29 \%$ of cases, but agree with human judgement in $70 \%$ of cases. While this requires more elaborate testing and rigorous methodology (Schütze, 1996; Schütze, 2005), the results tentatively indicate that highly discriminatory features could be useful in quality estimation of translations.

\section{Conclusion and Future Directions}

We have replicated and extended past work in identifying linguistic features that discriminate the source language of literary texts presented in English, perhaps through translation. Our replication of work by Lynch and Vogel (2012), using the same source data, method and feature types, ${ }^{11}$ yielded results which were a slight improvement on the original regarding the task of source language identification. Our reproduction of the experimental design on a fresh data set confirmed some signals of source language interference and showed others to be unreliable between data sets. This speaks to the need for still more extensive studies of this sort. We conclude that this method of source language detection on unseen texts of English from the literary genre is robust, and has potential for use in quality assessment for English prose. This work contributes to the emergent practice within the digital humanities of applying automatic text classification techniques to explore hypotheses.

Acknowledgements This research is conducted partly within the Centre for Global Intelligent Content (www.cngl.ie), funded by Science Foundation Ireland (12/CE/I2267).

1. Baroni, M. and Bernardini, S. "A new approach to the study of translationese: Machine-learning the difference between original and translated text". In: Literary and Linguistic Computing 21.3 (2006), pp. 259-274.

2. Becher, V. "Abandoning the notion of 'translation-inherent' explicitation. Against a dogma of translation studies". In: Across Languages and Cultures 11 (2010), pp. $1-28$.

3. Blum-Kulka, S. "Shifts of cohesion and coherence in translation". In: Interlingual and intercultural communication: Discourse and cognition in translation and second language acquisition studies 17 (1986), p. 35.

4. Degani, T., Prior, A., and Tokowicz, N. "Bidirectional transfer: The effect of sharing a translation”. In: J. of Cog. Psychology 23.1 (2011), pp. 18-28.

${ }^{11}$ Our current study employed POS trigrams, the original work used POS bigrams but not trigrams. 
5. Forsyth, R. S. and Lam, P. W. "Found in translation: To what extent is authorial discriminability preserved by translators?" In: Literary and Linguistic Computing 29.2 (2014), pp. 199-217.

6. Hall, M. et al. "The WEKA data mining software: an update". In: ACM SIGKDD explorations newsletter 11.1 (2009), pp. 10-18.

7. Ilisei, I. et al. "Identification of translationese: A machine learning approach". In: Computational Linguistics and Intelligent Text Processing. Springer, 2010, pp. 503-511.

8. Koppel, M. and Ordan, N. "Translationese and Its Dialects". In: Proceedings of the 49th Annual Meeting of the Association for Computational Linguistics: Human Language Technologies. Vol. 1. 2011, pp. 1318-1326.

9. Kurokawa, D., Goutte, C., and Isabelle, P. "Automatic Detection of Translated Text and its Impact on Machine Translation". In: Proceedings of the XII MT Summit, Ottawa, Ontario, Canada. AMTA. 2009.

10. Lembersky, G., Ordan, N., and Wintner, S. "Language Models for Machine Translation: Original vs. Translated Texts". In: Proc. of the 2011 Conf. on Empirical Methods in Natural Language Processing. 2011, pp. 363-374.

11. Lembersky, G., Ordan, N., and Wintner, S. "Adapting translation models to translationese improves SMT". In: Proceedings of the 13th Conference of the European Chapter of the Assoc. for Computational Linguistics, Avignon, France. 2012, p. 255.

12. Lynch, G. "A Supervised Learning Approach Towards Profiling the Preservation of Authorial Style in Literary Translations". In: Proc. 25th COLING. 2014, pp. 376-386.

13. Lynch, G. and Vogel, C. "Towards the Automatic Detection of the Source Language of a Literary Translation". In: Proc. 24th COLING (Posters). 2012, pp. 775-784.

14. Michalke, M. koRpus: An R Package for Text Analysis. (Version 0.04-40), last verified: 11.08.2014. 2013. URL: http://reaktanz.de/?c=hacking\&s=koRpus.

15. Schmid, H. "Probabilistic part-of-speech tagging using decision trees". In: Proceedings of international conference on new methods in language processing. Vol. 12. Manchester, UK. 1994, pp. 44-49.

16. Schütze, C. The Empirical Base of Linguistics: Grammaticality Judgements and Linguistic Methodology. University of Chicago Press, 1996.

17. Schütze, C. "Thinking About What We Are Asking Speakers to Do". In: Linguistic Evidence: Empirical, Theoretical and Computational Perspectives. Ed. by Kepsar, S. and Reis, M. Mouton De Gruyter, 2005, pp. 457-485.

18. van Halteren, H. "Source Language Markers in EUROPARL Translations". In: Proc. 22nd COLING. 2008, pp. 937-944.

19. Volansky, V., Ordan, N., and Wintner, S. "On the features of translationese". In: Literary and Linguistic Computing (2013). 